Rev. Elev. Méd. vét. Pays trop., 1979, 32 (2) : 131-134

\title{
Un foyer de botulisme de type $D$, lié à des modifications du milieu naturel, observé chez des pélicans (Pelecanus rufescens) du Sénégal (Petite Côte)
}

\author{
par M. P. DOUTRE
}

\begin{abstract}
RÉSUMÉ
Un foyer de botulisme de type D survenu chez des pélicans bruns est décrıt. L'isolement de la souche responsable a permis de déterminer son pouvoir toxinogène (DMM/sourıs : $10^{-7} \mathrm{ml}$ ). Le rejet d'eaux usées dans un marigot et la construction d'un barrage expliquent la création d'un environnement particulièrement favorable au développement de Clostridium botulınum. Ce foyer de botulisme survenu chez des oiseaux d'eau est le premier rapporté en Afrique de l'Ouest et Centrale. La mise en evidence du type D chez l'avifaune aquatique apparaît également comme un fait nouveau.
\end{abstract}

Le botulisme des oiseaux d'eau (Anatıdés, limicoles, etc...) a fait l'objet d'études nombreuses aux Etats-Unis et au Canada où périodiquement des hécatombes d'espèces variées (18) sont notées le long des voies de migrations (flyways) du continent nord-américain $(5,14,16)$. La maladie, connue sous les appellations de " limberneck » et de " duck-disease ", a revêtu une importance considérable pendant la première moitié du $\mathrm{xx}^{\mathrm{e}}$ siècle, époque durant laquelle des efforts considérables furent entrepris pour limıter les pertes.

Si l'on s'en tient aux années récentes, l'affection a été également signalée en Nouvelle-Zélande (15) mais aussi en Europe, tout particulièrement en Grande-Bretagne $(2,3,13)$ et au Pays-Bas (8). En Afrique, si l'on excepte un foyer rapporté en Afrique du Sud (9), aucune information n'existe à notre connaissance. Ce fait peut paraître surprenant, car la zone éthiopienne constitue, par ses réserves alimentaires et l'étendue

${ }^{(*)}$ Chef du service de Bactériologie au Laboratoire national de l'Elevage et de Recherches vétérinaires (I. S. R. A.), B. P. 2057 Dakar-Hann, République du Sénégal. de ses zones humides (delta du Séné gal, Borgou boucle du Niger, bassin Logone-Chari-Tchad, lacs de la vallée du Rift), un lieu d'hıvernage pour de nombreuses espèces paléarctiques. L'avifaune résidente est elle-même abondante et les rassemblements d'oiseaux souvent rencontrés sont comparables à ceux d'outre-Atlantique $^{\prime}$

Le présent article se propose de décrire un foyer de botulisme survenu chez des pélicans bruns (Pelecanus rufescens) demeurant sur un marigot aménagé, dans la concession d'un complexe hôtelier de la Petite Côte du Sénégal.

\section{TECHNIQUE D'ÉTUDE}

Un pélican brun est amené au Laboratoire à la mi-janvier 1979. L'animal est paralysé, mais encore en vie. Il appartient à une colonie de plusieurs dizaines d'individus où déjà des mortalités importantes ont été observées depuis plusieurs semaines. Les commémoratifs sont imprécis. Une analyse d'eau classique, effectuée par l'Institut Pasteur de Dakar, n'apporte pas 
d'éléments pouvant expliquer les accidents rencontrés.

L'autopsie n'offre qu'un parasitisme vermineux intestinal intense et un foie présentant à la section un suintement biliaire marqué.

Des ensemencements aérobies (sang du cœur, foie, contenu intestinal après enrichissement) et anaérobies (foie, contenu intestinal) sont effectués sur milieux ordinaires.

\section{RÉSULTATS}

Aucune des cultures en milieux aérobies ne permet d'isoler un germe pathogène quelconque (Pasteurella, Salmonella, etc...).

Par contre, les surnageants, dilués au 1/100, des cultures de foie et de contenu intestinal en milieu viande-foie, glucosé à 10 p. 100 , après 4 jours d'incubation à $37^{\circ} \mathrm{C}$ provoquent la mort de souris, en moins de $4 \mathrm{~h}(0,20 \mathrm{ml})$.

La culture mixte de foie, contaminée par un microcoque gram + , est seule conservée pour les manipulations ultérieures. Chauffée $10 \mathrm{mn}$ à $80^{\circ} \mathrm{C}$ et repiquée, elle fournit une subculture pure d'un Clostridium dont le surnageant, après 5 jours de culture, tue la souris à la dilution de $10^{-7} \mathrm{ml}$.

La toxinotypie botulique classique (100 DMM souris mélangées à une unité antitoxique de chacun des sérums antibotuliques $\mathrm{A}, \mathrm{B}, \mathrm{C}, \mathrm{D}$, $\mathrm{E}$; chaque mélange est inoculé à 5 souris sous un volume de $0,20 \mathrm{ml}$ ) montre que l'on se trouve en présence d'un foyer de botulisme aviaire de type $D$.

La souche isolée présente les caractères biochimiques habituels des souches de ce type précédemment rencontrées au Sénégal $(4,6)$. La toxine produite en milieu VF glucosé ne contient pas, ou renferme une quantité trop faible, de substance toxique convulsivante, soluble, non antigénique, insuffisante pour empêcher la sérotypie. Ce fait avait déjà été remarqué dans un cas récent (6), il en allait tout autrement de la première souche isolée (4).

\section{DISCUSSION}

\section{Enquête sur le terrain}

A la suite des résultats obtenus en laboratoire, un déplacement est accompli sur les lieux de résidence de la colonie de pélicans en cause. Les renseignements suivants sont recueillis :

- la colonie comptait dans le passé environ
300 sujets, actuellement on en dénombre une cinquantaine. Certaines semaines, 7 à 8 cadavres sont notés ou ramassés et ceci dès le début de la saison des pluies 1978 ,

- depuis la création du complexe hôtelier (6 ans), des aménagements ont été réalisés au niveau du marigot appelé improprement « lagune ». Un barrage a été édifié qui supprime désormais toute communication avec la mer. Une communication précaire est rétablie dans le sens marigot-océan en saison des pluies seulement (fig. 1),

- les eaux usées des cuisines, de l'eau à $100^{\circ} \mathrm{C}$ en provenance d'une buanderie, les lisiers d'une porcherie et les déchets d'un abattoir privé (porcs) sont déversés dans le marigot. Aucune station d'épuration n'existe actuellement et cette situation est fortement ressentie par la direction et le personnel très conscients du problème,

- la nappe liquide à proximité immédiate du barrage et de la route sur buses est de couleur vert sombre, chargée d'un phytoplancton abondant... A proximité du lieu de déversement des eaux usées, des bancs de plantes aquatiques prospèrent sur des hauts-fonds.

Des précisions sont recueillies sur l'évolution du niveau des eaux. Au cours des années passées, en raison des conditions climatiques exceptionnelles, le marigot s'assèchait très rapidement dans la zone de débouché des produits de déchets, lesquels stagnaient par flaques, entourées d'une boue sableuse durcie qui permettait même le passage de chevaux. Pour la première fois, en 1978 , les pluies d'hivernage normales, accrues par les précipitations inhabituelles de la petite saison des pluies, ont considérablement augmenté le volume des eaux qui en janvier demeurent encore à un niveau élevé, plus que suffisant pour assurer une communication permanente directe entre les points fréquentés par les pélicans- et l'arrivée des détritus divers.

Ces modifications apportées au milieu naturel suffisent pour expliquer l'apparition de ce foyer de botulisme aviaire, phénomène jusqu'à ce jour inconnu au Sénégal. L'échauffement d'eaux stagnantes, chargées en matières organiques, provoque un développement excessif de la flore algaire et microbienne où Clostridium botulinum trouve des conditions de croissance particulièrement favorables. Il est intéressant de souligner que le genre responsable a été mis en évjdence dans le foie et l'intestin et que l'on se trouve, tout au moins pour l'oiseau reçu au 


\section{L'EXPLOITATION DES PATURAGES DE SAVANE}

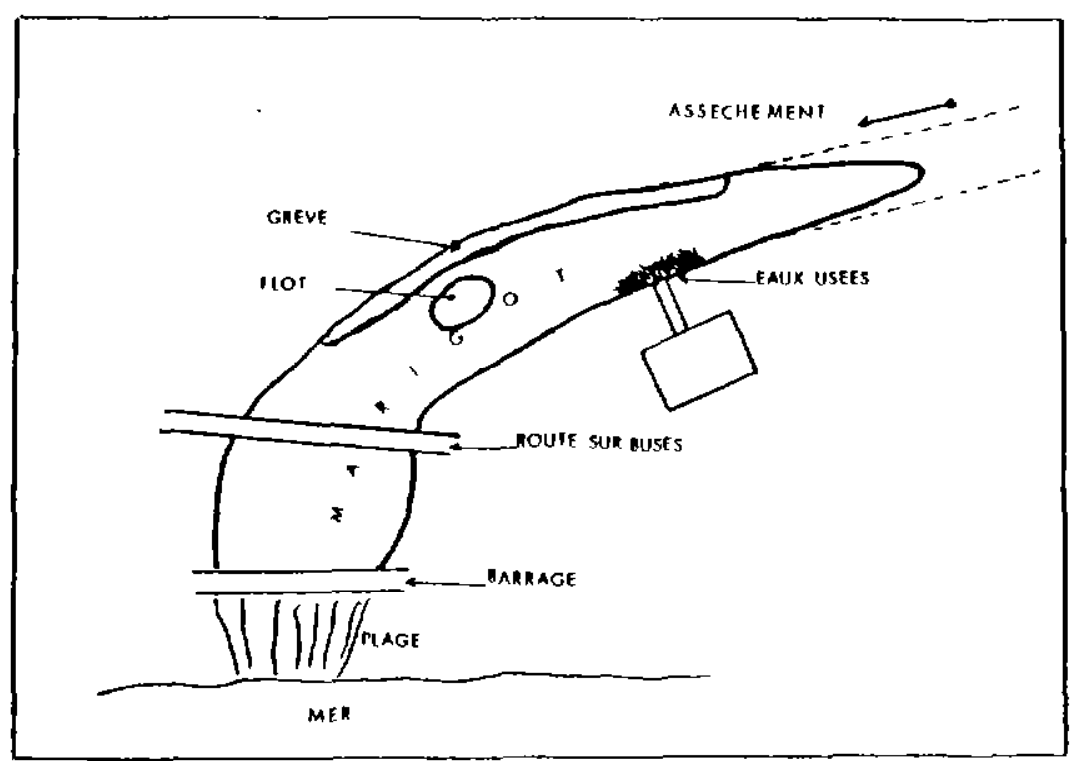

Fig. I. -Schéma du marigot (longueur à la mi-janvier:200 m,

en fin de saison des plutes:2 km).

Laboratoire, en présence d'un cas de toxi-infection et non d'intoxination (la littérature rapporte le plus souvent des cas d'intoxination chez les oișeaux d'eau) $(1,5,8,9,10,11)$.

Les mesures préconisées, pour prévenir le retour d'une telle situation, découlent de l'analyse des causes. En premier lieu, il est indispensable d'assurer le libre accès du marigot à la mer, ensuite le rejet dans ses eaux de tout élément organique polluant est formellement à proscrire. La construction d'une station d'épuration s'avère ainsi impérative.
Sur le plan bactériologique, les études passées nous montrent que le botulisme des oiseaux d'eau est principalement occasionné par le type $\mathrm{C}$ de Clostridium botulinum. Le sous-type alpha du type $\mathrm{C}$, dont l'existence est désormais contestée par JANSEN (12), avait été découvert en Amérique du Nord lors des enzooties de "limberneck». Le type pisciaire E est souvent cause de mortalités chez les mouettes et les sternes. Mais à notre connaissance, aucun cas de botulisme de type D n'avait encore été rapporté chez l'avifaune aquatique.

\section{SLMMARY}

An outbreak of botulism type $D$, caused by changes in natural environment, in pink-backed pelicans (Pelecanus rufescens) (Petite Côte, Sénégal).

An outbreak of botulism type D which occurred in pink-backed pelicans is described. The isolation of the strain involved has allowed to determine its toxinogenic property (DMM : $10^{-7} \mathrm{ml}$ ). The running of waste waters in a marigot and the bulding of a dam explain the development of an environment particularly favourable to the growing of Clostridium botulinum. This waterfowl outbreak of botulism is the first reported in Western and Central Africa. The demonstration of type $D$ in waterfowl appears also as a new fact.

\section{RESUMEN}

Un foco de botulismo de tipo D, ligado con modificaciones del medio natural, observado en pelícanos (Pelecanus rufescens) de Senegal (Pequena Costa)

Se describe un foco de botulismo de tipo B ocurrido en los pelícanos pardos. El aislamiento de la cepa responsable permitió determinar su poder toxinogeno (DMM/ratón : $10^{-7} \mathrm{ml}$ ). 
El echamiento de aguas residuales en un brazo de río y la construcción de un embalse explican la creación de un medio ambiente particularmente favorable al desarrollo de Clostridium botulinum. Este foco de botulismo ocurrido en las aves de agua es el primer notado en Africa del oeste y del Centro. EI evidenciar del tipo $\mathrm{D}$ en las aves acuáticas parece también nuevo.

\section{BIBLIOGRAPHIE}

1. BELL (J. F), SCIPLE (G. W.), HUBERT (A. A.). A micro-environment concept of the epizoology of avian botulism. $J$. Wildl. Mgmt., 1955, 19 : 352-357.

2. BLANDFORD (T. B.), ROBERTS (T. A.), ASHTON (W. L. G.). Losses from botulism in mallard duck and other waterfowl. Vet. Rec., 1969, 85 (20) : 541-543.

3. BORLAND (E. D.), MORYSON (C. J.), SMITH (G. R.). Avian botulism and the high prevalence of Clostridium botulinum in the Norfolk Broads. Vet. Rec., 1977, 100 (6) : 106-109.

4. CHAMBRON (J.), MARTEL (J. L), DOUTRE (M. P.). Le botulisme équin au Sénégal. Premier isolement de Clostridium botulinum type D. Rev. Elev. Méd. vét. Pays trop., 1971, 24 (1) : 1-17.

5. DAVIS (J. W.), ANDERSON (R. C.), KARSSTAD (L.), TRAINER (D. O.). Infectious and parasitic diseases of wild birds. Ames, lowa, U. S. A., The Iowa State Univ. Press, 1971.

6. DOUTRE (M. P.), TOURE (B.). A propos d'un nouveau cas de botulisme hydrique de type $D$ survenu au Sénégal. Considérations étiopathogéniques. Rev. Elev. Méd. vét. Pays trop., 1978, 31 (4) : 411-415.

7. GRETILLAT (S.), MOREL (P.). Nématodes et trématodes trouvés chez un pélican (Pelecanus onocrotalus L.) au Sénégal. Bull. Inst. Fr. Afr. Noire, 1961, $23(2): 423-437$.

8. HAAGSMA (J.), OVER (H. J.), SMITH (T.), HOEKSTRA (J.). Botulism in waterfowl in the Netherlands in 1970. Nether. J. vet. Sci, 1972, 5 : 1234.

9. HAY (C. M. E.), VAN DER MADE (H. N.), KNOETZE (P. C.). Isolation of Clostriditum botuli- num type $\mathrm{C}$ from an outbreak of botulism in wild geese. J. S. Afr. vet. Ass., 1973, $44: 53-56$.

10. HUNTER (B. F.). Ecology of waterfowl botulism toxin production. Trans. N. Amer. Wildl. Nat. Resources Conf., $1970: 64-72$.

11. HUNTER (B. F.), CLARK (W. E.), PERKINS (P. J.), COLEMAN (P. R.). Applied botulism research including management recommendations. Sacramento, California, Calıf. Dept. Fish \& Game, 1970.

12. JANSEN (B. C.), KNOETZE (P. C.). The taxonomic position of Clostridium botulinum type C. Onderstepoort J. vet. Res., 1977, 44 (2) : 53-54.

13. KEYMER (I. F.), SMITH (G. R.), ROBERTS (T. A.), HEANEY (S. I.), HIBBERD (D. J.). Botulism as a factor in waterfowl mortality at St. James Park, London. Vet. Rec., 1972, 90 : 111-114.

14. LEWIS (K. H.), CASSEL (K. Jr.). Botulısm. Proc. of a Symp., jan. 13-15, 1964. U. S. Cincinatti, Ohio, Dept. of Hlth Education \& Welfare, Public Hlth Serv., dec. 1964.

15. MARTINOVICH (D.), CARTER (M. E.), WOODHOUSE (D. A.), McCAUSLAND (I. P.). An outbreak of botulism in wild waterfowl in New Zealand. N. Z. vet. J., 1972, $20: 61-65$.

16. MIERZEJEWSKI (J.). Botulism zwierzat domowych j dzikich. Varsovie, Panstwowe Wydawnictwo rolnicze i lesne, 1969.

17. SCHWARTZ (L.), SMART (G.), Control of botulism in wildfowl. J. Am. vet. Med. Ass., 1963, $143: 163$.

18. SMITH (L.). Botulism. The organism, its toxins, the disease. Springfield, 111. U. S A. Charles C. Thomas éd., 1977. 